\title{
Bipolar Quantum Logic Gates and Quantum Cellular Combinatorics-A Logical Extension to Quantum Entanglement
}

\author{
Wen-Ran Zhang \\ Department of Computer Science, College of Engineering and Information Technology, \\ Georgia Southern University, Statesboro, USA \\ Email: wrzhang@georgiasouthern.edu
}

Received April 15, 2013; revised May 18, 2013; accepted May 28, 2013

Copyright (C) 2013 Wen-Ran Zhang. This is an open access article distributed under the Creative Commons Attribution License, which permits unrestricted use, distribution, and reproduction in any medium, provided the original work is properly cited.

\begin{abstract}
Based on bipolar dynamic logic (BDL) and bipolar quantum linear algebra (BQLA) this work introduces bipolar quantum logic gates and quantum cellular combinatorics with a logical interpretation to quantum entanglement. It is shown that: 1) BDL leads to logically definable causality and generic particle-antiparticle bipolar quantum entanglement; 2) BQLA makes composite atom-atom bipolar quantum entanglement reachable. Certain logical equivalence is identified between the new interpretation and established ones. A logical reversibility theorem is presented for ubiquitous quantum computing. Physical reversibility is briefly discussed. It is shown that a bipolar matrix can be either a modular generalization of a quantum logic gate matrix or a cellular connectivity matrix. Based on this observation, a scalable graph theory of quantum cellular combinatorics is proposed. It is contended that this work constitutes an equilibrium-based logical extension to Bohr's particle-wave complementarity principle, Bohm's wave function and Bell's theorem. In the meantime, it is suggested that the result may also serve as a resolution, rather than a falsification, to the EPR paradox and, therefore, a equilibrium-based logical unification of local realism and quantum non-locality.
\end{abstract}

Keywords: Bipolar Causal Sets; Logically Definable Causality; Basis State Quantum Entanglement; Generic and Composite Entanglement; Bipolar Equilibrium; Bipolar Quantum Logic Gates; Quantum Cellular Combinatorics

\section{Introduction}

Aristotle's causality principle denotes a necessary relationship between one event called cause and another event called effect which is the direct consequence of the cause. Without making clear the cause-effect relation any scientific theory can be said incomplete. But in more than 2300 years, all truth-based systems failed to provide logically definable causality with regularity. The dilemma was recognized and reiterated by a number of legendary figures, notably, by David Hume-a founder of modern empiricism who challenged Aristotle's causality principle [1], Niels Bohr - a father figure of quantum mechanics who asserted that quantum causality is unattainable [2], and Lotfi Zadeh-founder of fuzzy logic who bluntly asserted that "Causality is undefinable" [3].

In quantum mechanics, Bell's theorem [4] has been considered by many as a falsification of the EinsteinPodolsky-Rosen (EPR) argument [5]. It has led to a num- ber of experiments that statistically support Bohr's interpretation of quantum entanglement-the "spooky action in a distance" so-called by Einstein. But logically definable causality for quantum entanglement remains a mystery until today and the validity of Bell inequalities is still been questioned with experimental loopholes [6].

Niels Bohr was the first to bring YinYang into quantum theory for his particle-wave complementarity principle. When he was awarded the Order of the Elephant by the Danish government in 1947, he designed his own coat of arms which featured in the center a YinYang logo (Taiji symbol) with the Latin motto "contraria sunt complementa" or "opposites are complementary" (Figure 1).

While quantum mechanics recognized particle-wave complementarity, it stopped short of identifying the essence of YinYang bipolar coexistence. Without bipolarity any complementarity is less fundamental due to the missing "opposites" (see Figure 2). On the other hand, if bipolar equilibrium is the most fundamental form of 


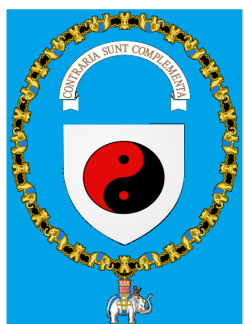

Figure 1. Bohr's Coat of Arms (Creative Commons file by GJo, 3/8/2010, Source File: Royal Coat of Arms of Denmark. svg).

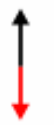

(a)

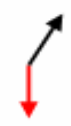

(b)

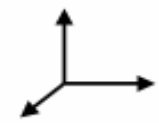

(c)

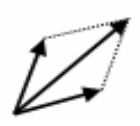

(d)
Figure 2. Fundamental and non-fundamental complementtarities. (a) Fundamental; (b)-(d) Non-fundamental.

equilibrium, any multidimensional model in spacetime geometry cannot be most fundamental. In brief, actionreaction, particle-antiparticle, input-output, negativepositive energies, or the Yin and Yang of nature in general constitute the most fundamental opposites, but man and woman, space and time, particle and wave, truth and falsity are not exactly bipolar opposites. This could be the reason why Bohr believed that a causal description of a quantum process cannot be attained and quantum mechanics has to content itself with particle-wave complementary descriptions [2]. It may also be the reason why Bohr's Copenhagen interpretation was challenged by the EPR thought experiment, Bohm's wave function [7] was dismissed by Einstein as "too cheap" [8, p. 341], and Bell's theorem stopped short of reaching logically definable quantum causality and had to rely on probability and statistical measures that is still been questioned.

Actually, following David Bohm [7], Bell relied on two entangled qubits in spin or polarization. In his theorem, spin-up $(+)$ or spin-down $(-)$ were used as essential concepts [4]. But Bell, like Bohr and Bohm, stopped short of developing an equilibrium-based dynamic logical system to account for the equilibrium and non-equilibrium states of negative-positive energies - the most fundamental Yin and Yang of nature.

While quantum computing has made progress, without quantum causality, the ubiquitous effects of quantum entanglement so far remains a mystery. For instance, the intrinsic relationships between quantum circuitry and biocellular networks are unknown. As a result, quantum cellular combinatorics remains an untouched gap between quantum computing, biological computing and human intelligence.

Is quantum causality logically definable? Further extending Bohr's particle-wave complementarity principle and Bell's theorem, this work introduces an equilib- rium-based logical interpretation to quantum causality and entanglement for quantum logic gate design and quantum cellular combinatorics. First, bipolar dynamic logic (BDL) and bipolar quantum linear algebra (BQLA) are introduced. While BDL supports logically definable causality and generic quantum entanglement, BQLA provides algebraic causality and composite quantum entanglement. The two levels of causality and entanglements enable the generalization of quantum logic gate matrix from unipolar truth-based domain to bipolar equilibriumbased domain. This generalization presents a unification of digital computing, quantum computing and bio-cellular computing with a possible resolution to the EPR paradox. A scalable graph theory of quantum cellular combinatorics is introduced for ubiquitous quantum computing and quantum intelligence.

This paper is organized into six sections. Section 2 introduces BDL and BQLA. Section 3 discusses bipolar quantum (logic) gates and quantum causality with two levels of entanglement. Section 4 extends bipolar quantum gates to quantum cellular combinatorics. Section 5 presents an analysis and discussion. Section 6 draws a few conclusions.

\section{Quantum Causality Is Logically Definable}

\subsection{Bipolar Dynamic Logic (BDL)}

YinYang bipolar dynamic logic (BDL) presents an equilibrium-based extension to quantum computing [9-12]. $\mathrm{BDL}$ is based on bipolar sets whose elements are bipolar agents such as dipoles, particle-antiparticle pairs, nature's action-reaction objects, genomic repression-activation capacities, social competition-cooperation relations, inputoutput of any system, self-negation and self-assertion abilities in mental health, in general, the negative and positive energies of nature (Figure 3). This ontological claim positioned BDL in the context of logically definable causality for ubiquitous quantum computing.

BDL is defined on

$B_{1}=\{-1,0\} \times\{0,+1\}=\{(0,0),(0,+1),(-1,0),(-1,+1)\} \quad$ a bipolar quantum lattice in YinYang bipolar geometry as shown in Figure 4. The four values of $B_{1}$ form a bipolar causal set which stand, respectively, for eternal equilibrium $(0,0)$, non-equilibrium $(-1,0)$, non-equilibrium $(0,+1)$; equilibrium $(-1,+1)$. Evidently, each bipolar element can be used to code two bits of binary information (or one bit with an OR or AND operation of the two poles in absolute values). Figure 5 illustrates bipolar interaction and entanglement.

Equations (1)-(12) in Table 1 provide the basic operations of BDL. The laws in Table 2 hold on BDL. Bipolar universal modus ponens (BUMP) is listed in Table 3 which logically defines equilibrium-based bipolar causality. Truth tables of BDL are presented in Table 4. In BDL $\oplus$ and $\oplus^{-}$are "balancers"; $\varnothing$ and $\otimes$ are intuitive "oscilla- 


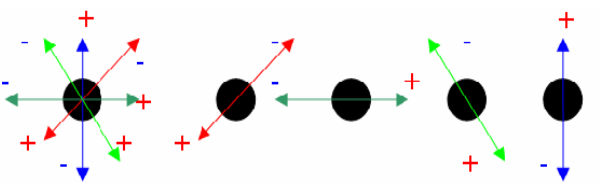

Figure 3. Multidimensional equilibrium or non-equilibrium deconstructed to bipolar equilibria/non-equilibria.

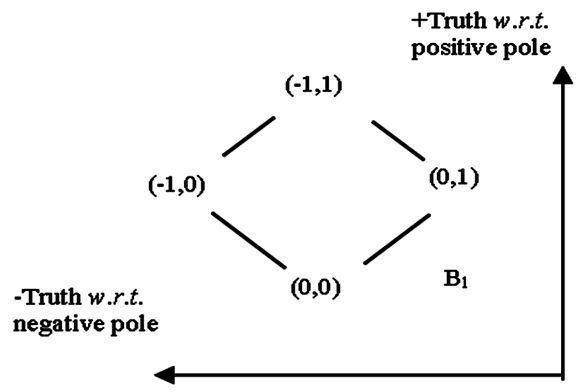

Figure 4. Hasse diagram of $B_{1}$ in bipolar geometry.

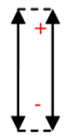

(a)

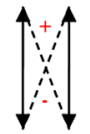

(b)

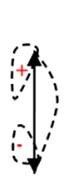

(c)

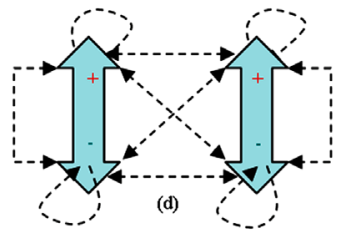

Figure 5. Bipolar relativity: (a) Linear interaction; (b) Crosspole non-linear interaction; (d) Oscillation; (e) Bipolar entanglement.

Table 1. Yin Yang Bipolar Dynamic Logic (BDL).

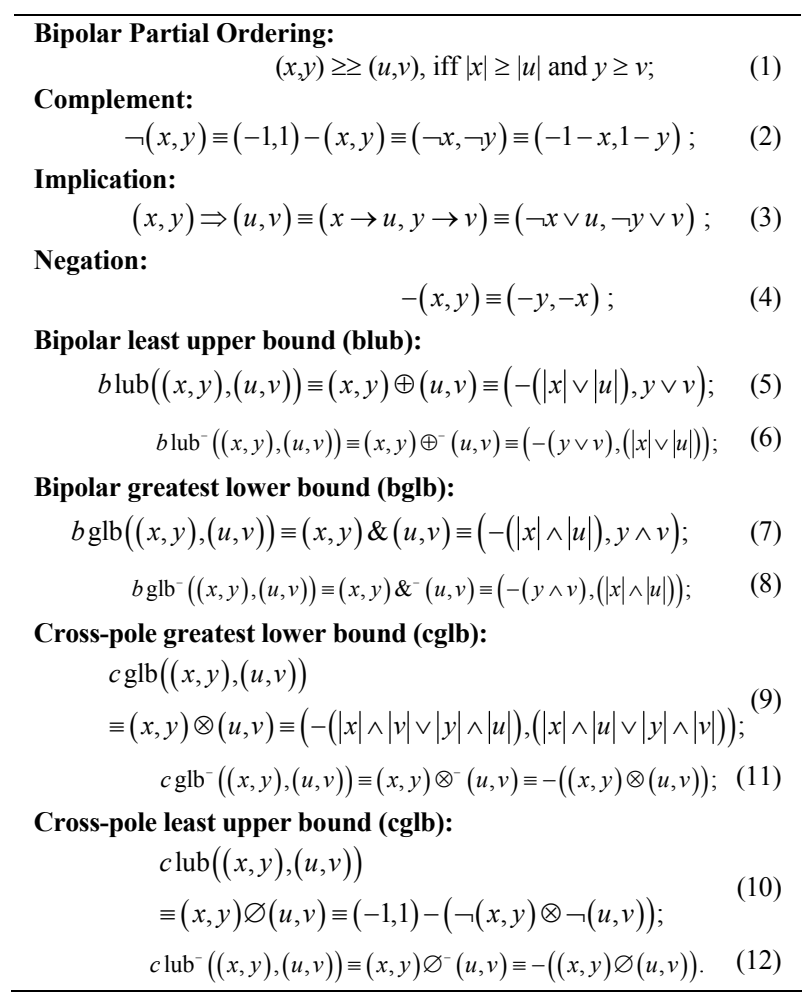

(Note: The use of $|x|$ in this paper is for explicit bipolarity only).
Table 2. Laws of bipolar equilibrium/non-equilibrium.

\begin{tabular}{lc}
\hline Excluded Middle & $(x, y) \oplus(x, y) \equiv(-1,1) ;$ \\
& $(x, y) \oplus^{-} \neg(x, y) \equiv(-1,1) ;$ \\
\hline No contradiction & $((x, y) \& \neg(x, y)) \equiv(-1,1) ;$ \\
& $\neg\left((x, y) \&^{-} \neg(x, y)\right) \equiv(-1,1) ;$ \\
\hline Linear Bipolar & $\neg((a, b) \&(c, d)) \equiv \neg(a, b) \oplus \neg(c, d) ;$ \\
DeMorgan’s Laws & $\neg((a, b) \oplus(c, d)) \equiv \neg(a, b) \& \neg(c, d) ;$ \\
& $\neg\left((a, b) \&^{-}(c, d)\right) \equiv \neg(a, b) \oplus^{-} \neg(c, d) ;$ \\
& $\neg\left((a, b) \oplus^{-}(c, d)\right) \equiv \neg(a, b) \&^{-} \neg(c, d) ;$ \\
Non-Linear Bipolar & $\neg((a, b) \varnothing(c, d)) \equiv \neg(a, b) \otimes \neg(c, d) ;$ \\
DeMorgan’s & $\neg((a, b) \otimes-(c, d)) \equiv \neg(a, b) \varnothing^{-} \neg(c, d) ;$ \\
Laws & $\neg\left((a, b) \varnothing^{-}(c, d)\right) \equiv \neg(a, b) \otimes-\neg(c, d) ;$ \\
\hline
\end{tabular}

Table 3. Bipolar Universal Modus Ponens (BUMP).

$\forall \phi=\left(\phi^{-}, \phi^{+}\right), \varphi=\left(\varphi^{-}, \varphi^{+}\right), \psi=\left(\psi^{-}, \psi^{+}\right), \quad \chi=\left(\chi^{-}, \chi^{+}\right) \in B_{1}$,

$$
\begin{gathered}
{[(\phi \Rightarrow \varphi) \&(\psi \Rightarrow \chi)] \Rightarrow[(\phi * \psi) \Rightarrow(\varphi * \chi)] ; \boldsymbol{O R}} \\
(\phi \Leftrightarrow \varphi) \Rightarrow(\phi * \psi) \Leftrightarrow(\varphi * \psi)
\end{gathered}
$$

Two-fold universal instantiation:

1) Operator instantiation: * as a universal operator can be bound to $\&, \oplus, \&^{-}, \oplus^{-}, \otimes, \varnothing, \otimes^{-}, \varnothing^{-} .(\phi \Rightarrow \varphi)$ is designated (bipolar true $(-1,+1) ;\left(\left(\phi^{-}, \phi^{+}\right) *\left(\psi^{-}, \psi^{+}\right)\right)$is undesignated.

2) Variable instantiation:

$\forall x,\left(\phi^{-}, \phi^{+}\right)(x) \Rightarrow\left(\varphi^{-}, \varphi^{+}\right)(x) ;\left(\phi^{-}, \phi^{+}\right)(A) ; \therefore\left(\varphi^{-}, \varphi^{+}\right)(A)$.

tors"; $\varnothing^{-}$and $\otimes^{-}$are counter-intuitive "oscillators"; \& and $\&^{-}$are "minimizers." The linear, cross-pole, bipolar fusion, fission, oscillation, interaction and entanglement properties are depicted in Figure 5.

Thus, BDL presents a causal logic or a causal set approach to quantum computing. The causal set hypothesis is originally a quest for quantum gravity [13]. As a settheoretic model, however, causal sets did not go beyond classical truth-based set theory to reach logically definable quantum causality until bipolar sets and BDL.

\subsection{Bipolar Quantum Linear Algebra (BQLA)}

The bipolar lattice $B_{1}=\{-1,0\} \times\{0,1\}$ can be naturally extended to the bipolar fuzzy lattice $B_{F}=[-1,0] \times[0,1]$ and real valued bipolar lattice $B_{\infty}=[-\infty, 0] \times[0,+\infty] . B_{1}$ and $B_{F}$ are bounded complemented unit square lattices, respectively; $B_{\infty}$ is unbounded. $\forall(x, y),(u, v) \in B_{\infty}$, Equations (13) and (14) define two algebraic operations.

Tensor Bipolar Multiplication:

$$
(x, y) \times(u, v) \equiv(x v+y u, x u+y v) ;
$$

Bipolar Addition:

$$
(x, y)+(u, v) \equiv(x+u, y+v) .
$$


Table 4. Truth tables.

(a)

\begin{tabular}{ccccc}
\hline $\boldsymbol{\&}$ & $\mathbf{( 0 , 0 )}$ & $\mathbf{( - 1 , 0 )}$ & $\mathbf{( 0 , 1 )}$ & $\mathbf{( - 1 , 1 )}$ \\
\hline $\mathbf{( 0 , 0 )}$ & $(0,0)$ & $(0,0)$ & $(0,0)$ & $(0,0)$ \\
$\mathbf{( - 1 , 0 )}$ & $(0,0)$ & $(-1,0)$ & $(0,0)$ & $(-1,0)$ \\
$(\mathbf{0 , 1})$ & $(0,0)$ & $(0,0)$ & $(0,1)$ & $(0,1)$ \\
$(-\mathbf{1 , 1})$ & $(0,0)$ & $(-1,0)$ & $(0,1)$ & $(-1,1)$ \\
\hline
\end{tabular}

(b)

\begin{tabular}{ccccc}
\hline$\oplus$ & $\mathbf{( 0 , 0 )}$ & $\mathbf{( - 1 , 0 )}$ & $\mathbf{( 0 , 1 )}$ & $\mathbf{( - 1 , 1 )}$ \\
\hline $\mathbf{( 0 , 0 )}$ & $(0,0)$ & $(-1,0)$ & $(0,1)$ & $(-1,1)$ \\
$\mathbf{( - 1 , 0 )}$ & $(-1,0)$ & $(-1,0)$ & $(-1,1)$ & $(-1,1)$ \\
$\mathbf{( 0 , 1 )}$ & $(0,1)$ & $(-1,1)$ & $(0,1)$ & $(-1,1)$ \\
$(-\mathbf{1 , 1 )}$ & $(-1,1)$ & $(-1,1)$ & $(-1,1)$ & $(-1,1)$ \\
\hline
\end{tabular}

(c)

\begin{tabular}{ccccc}
\hline$\otimes$ & $\mathbf{( 0 , 0 )}$ & $\mathbf{( - 1 , 0 )}$ & $\mathbf{( 0 , 1 )}$ & $\mathbf{( - 1 , \mathbf { 1 } )}$ \\
\hline $\mathbf{( 0 , 0 )}$ & $(0,0)$ & $(0,0)$ & $(0,0)$ & $(0,0)$ \\
$\mathbf{( - 1 , 0 )}$ & $(0,0)$ & $(0,1)$ & $(-1,0)$ & $(-1,1)$ \\
$\mathbf{( 0 , 1 )}$ & $(0,0)$ & $(-1,0)$ & $(0,1)$ & $(-1,1)$ \\
$\mathbf{( - 1 , 1 )}$ & $(0,0)$ & $(-1,1)$ & $(-1,1)$ & $(-1,1)$ \\
\hline
\end{tabular}

(d)

\begin{tabular}{ccccc}
\hline$\varnothing$ & $\mathbf{( 0 , 0 )}$ & $\mathbf{( - 1 , 0 )}$ & $\mathbf{( 0 , 1 )}$ & $\mathbf{( - 1 , 1 )}$ \\
\hline $\mathbf{( 0 , 0 )}$ & $(0,0)$ & $(0,0)$ & $(0,0)$ & $(-1,1)$ \\
$\mathbf{( - 1 , 0 )}$ & $(0,0)$ & $(-1,0)$ & $(0,1)$ & $(-1,1)$ \\
$\mathbf{( 0 , 1 )}$ & $(0,0)$ & $(0,1)$ & $(-1,0)$ & $(-1,1)$ \\
$(-\mathbf{1}, \mathbf{1})$ & $(-1,1)$ & $(-1,1)$ & $(-1,1)$ & $(-1,1)$ \\
\hline
\end{tabular}

(e)

\begin{tabular}{ccccc}
\hline $\boldsymbol{\&}^{-}$ & $\mathbf{( 0 , 0 )}$ & $\mathbf{( - 1 , 0 )}$ & $\mathbf{( 0 , 1 )}$ & $\mathbf{( - 1 , 1 )}$ \\
\hline $\mathbf{( 0 , 0 )}$ & $(0,0)$ & $(0,0)$ & $(0,0)$ & $(0,0)$ \\
$(\mathbf{- 1 , 0 )}$ & $(0,0)$ & $(0,1)$ & $(0,0)$ & $(0,1)$ \\
$(\mathbf{0 , 1 )}$ & $(0,0)$ & $(0,0)$ & $(-1,0)$ & $(-1,0)$ \\
$(-\mathbf{1}, \mathbf{1})$ & $(0,0)$ & $(0,1)$ & $(-1,0)$ & $(-1,1)$ \\
\hline
\end{tabular}

(f)

\begin{tabular}{ccccc}
\hline$\oplus^{-}$ & $\mathbf{( 0 , 0 )}$ & $\mathbf{( - 1 , 0 )}$ & $\mathbf{( 0 , 1 )}$ & $\mathbf{( - 1 , 1 )}$ \\
\hline $\mathbf{( 0 , 0 )}$ & $(0,0)$ & $(0,1)$ & $(-1,0)$ & $(-1,1)$ \\
$\mathbf{( - 1 , 0 )}$ & $(0,1)$ & $(0,1)$ & $(-1,1)$ & $(-1,1)$ \\
$\mathbf{( 0 , 1 )}$ & $(-1,0)$ & $(-1,1)$ & $(-1,0)$ & $(-1,1)$ \\
$(-\mathbf{1 , 1})$ & $(-1,1)$ & $(-1,1)$ & $(-1,1)$ & $(-1,1)$ \\
\hline
\end{tabular}

(g)

\begin{tabular}{ccccc}
\hline$\Theta^{-}$ & $\mathbf{( 0 , 0 )}$ & $\mathbf{( - 1 , 0 )}$ & $\mathbf{( 0 , 1 )}$ & $\mathbf{( - 1 , 1 )}$ \\
\hline $\mathbf{( 0 , 0 )}$ & $(0,0)$ & $(0,0)$ & $(0,0)$ & $(0,0)$ \\
$\mathbf{( - 1 , 0 )}$ & $(0,0)$ & $(-1,0)$ & $(0,1)$ & $(-1,1)$ \\
$(\mathbf{0 , 1 )}$ & $(0,0)$ & $(0,1)$ & $(-1,0)$ & $(-1,1)$ \\
$\mathbf{( - 1 , 1 )}$ & $(0,0)$ & $(-1,1)$ & $(-1,1)$ & $(-1,1)$ \\
\hline
\end{tabular}

(h)

\begin{tabular}{ccccc}
\hline $\boldsymbol{\varnothing}^{-}$ & $\mathbf{( 0 , 0 )}$ & $\mathbf{( - 1 , 0 )}$ & $\mathbf{( 0 , 1 )}$ & $\mathbf{( - 1 , 1 )}$ \\
\hline $\mathbf{( 0 , 0 )}$ & $(0,0)$ & $(0,0)$ & $(0,0)$ & $(-1,1)$ \\
$\mathbf{( - 1 , 0 )}$ & $(0,0)$ & $(0,1)$ & $(-1,0)$ & $(-1,1)$ \\
$\mathbf{( 0 , 1 )}$ & $(0,0)$ & $(-1,0)$ & $(0,1)$ & $(-1,1)$ \\
$\mathbf{( - 1 , 1 )}$ & $(-1,1)$ & $(-1,1)$ & $(-1,1)$ & $(-1,1)$ \\
\hline
\end{tabular}

(i)

\begin{tabular}{ccccc}
\hline $\mathbf{( x , y )}$ & $\mathbf{( 0 , 0 )}$ & $\mathbf{( - 1 , 0 )}$ & $\mathbf{( 0 , 1 )}$ & $\mathbf{( - 1 , 1 )}$ \\
\hline$\neg(\mathbf{x}, \mathbf{y})$ & $(-1,+1)$ & $(0,1)$ & $(-1,0)$ & $(0,0)$ \\
$-(\mathbf{x}, \mathbf{y})$ & $(0,0)$ & $(0,1)$ & $(-1,0)$ & $(-1,-1)$ \\
\hline
\end{tabular}

In Equation (13), $\times$ is a cross-pole multiplication operator with the infused non-linear bipolar tensor semantics of $--=+,-+=+-=1$, and $++=+$; + in Equation (14) is a linear bipolar addition or fusion operator. With the logical and algebraic operations, classical linear algebra is naturally extended to an equilibrium-based bipolar quantum linear algebra (BQLA) with bipolar fusion, fission, diffusion, interaction, oscillation, annihilation, and quantum entanglement properties. These properties enable physical or biological agents to interact through bipolar quantum fields such as atom-atom, cell-cell, heart-heart, heart-brain, brain-brain, organ-organ, and genome-genome bio- electromagnetic quantum fields as well as biochemical pathways in energy equilibrium or non-equilibrium. Thus, the properties lead to the inception of bipolar atom and a new philosophy $[14,15]$.

Based on Equations (13) and (14), given input vector $E$ and a bipolar matrix $M$ we have Equation (15). While $E$ is the input vector to a dynamic system characterized with the connectivity matrix $M, V$ is the result column bipolar vector.

$$
V=M \times E
$$

$M$ can actually be a bipolar generalization of a quantum gate matrix. The generalization is made possible by BDL and BQLA. With the generalization we have the following definition of energy and equilibrium.

The energy of a bipolar element or bipolar matrix is the absolute total of the negative and positive sides.

For instance, the absolute energy of a bipolar element $(-0.5,+0.1)$ can be defined as

$$
|\varepsilon|(-0.5,0.1) \equiv|\varepsilon|(-0.1,0.5)=|-0.1|+0.5=0.6 \text {. }
$$

Bipolar Energy Conservational Quantum Gate. If the energy of every row and every column of a bipolar decimal matrix $\mathrm{M}$ in Eq. (15) always adds up to 1.0, we call $\mathrm{M}$ a bipolar energy conservational quantum gate matrix.

Law of Bipolar Equilibrium or Symmetry: $\forall V, M$, $E$, if $M$ is an energy conservational quantum gate matrix, $V=M \times E$ satisfies Equation (16).

$$
|\varepsilon|(V)=|\varepsilon|(M \times E) \equiv|\varepsilon|(E)
$$

Evidently, any integer unitary quantum gate matrix must be energy conservational. Therefore, a unitary quantum gate can be deemed part of energy conservation or equilibrium for reversibility.

\section{Bipolar Quantum Gates and Quantum Entanglement}

\subsection{Logical Examples}

Similar to classical linear matrix, the matrix $M$ in Equation (15) as a causal set can be used to characterize a bipolar quantum gate. Using BDL and BQLA we design two such gates to illustrate the basic ideas in the follow- 
ing.

Gate 1. B-NOT Gate (Bipolar NOT Gate):

Graph form:

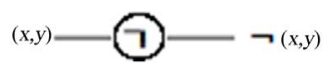

Bipolar Matrix $\mathrm{M}_{1}$ (Satisfies Equation (16)):

$$
M_{1}=\left[\begin{array}{rrrr}
(0,0) & (0,0) & (0,0) & (0,1) \\
(0,0) & (0,0) & (0,1) & (0,0) \\
(0,0) & (0,1) & (0,0) & (0,0) \\
(0,1) & (0,0) & (0,0) & (0,0)
\end{array}\right]
$$

In simplified unipolar forms

$$
M_{1}=\left[\begin{array}{llll}
0 & 0 & 0 & 1 \\
0 & 0 & 1 & 0 \\
0 & 1 & 0 & 0 \\
1 & 0 & 0 & 0
\end{array}\right]
$$

Interestingly, if we regard the ordered bipolar causal set $\{(0,0),(0,1),(-1,0),(-1,1)\}$ as equivalent to the set of basis states $\{|00\rangle,|01\rangle,|10\rangle,|11\rangle\}$ we have

$$
M_{1} \times\left[\begin{array}{c}
(0,0) \\
(0,1) \\
(-1,0) \\
(-1,1)
\end{array}\right] \equiv \neg\left[\begin{array}{c}
(0,0) \\
(0,1) \\
(-1,0) \\
(-1,1)
\end{array}\right]=\left[\begin{array}{c}
(-1,1) \\
(-1,0) \\
(0,1) \\
(0,0)
\end{array}\right]
$$

which would be equivalent to

$$
\neg\left[\begin{array}{c}
|00\rangle \\
|01\rangle \\
|10\rangle \\
|11\rangle
\end{array}\right]=\left[\begin{array}{c}
|11\rangle \\
|10\rangle \\
|01\rangle \\
|00\rangle
\end{array}\right]
$$

Noticeably, the matrix $M_{1}$ is similar to controlled NOT gate (C-NOT or XOR gate) but fundamentally different. Using bipolar quantum linear algebra, we can apply the quantum gate matrix directly to an input bipolar column vector and do not have to go through the eigenvalues of each basis state.

Different from unipolar quantum computing, bipolar quantum logic provides bipolar negation operation in addition to the B-NOT operator.

Gate 2. B-NEG Gate (Bipolar Negation Gate):

Graph form:

$$
(x, y)-\Theta--(x, y)
$$

Bipolar Matrix $\mathrm{M}_{2}$ (Satisfies Equation (16)):

$$
M_{2}=\left[\begin{array}{cccc}
(-1,0) & (0,0) & (0,0) & (0,0) \\
(0,0) & (-1,0) & (0,0) & (0,0) \\
(0,0) & (0,0) & (-1,0) & (0,0) \\
(0,0) & (0,0) & (0,0) & (-1,0)
\end{array}\right]
$$

or in simplified form

$$
M_{2}=\left[\begin{array}{cccc}
-1 & 0 & 0 & 0 \\
0 & -1 & 0 & 0 \\
0 & 0 & -1 & 0 \\
0 & 0 & 0 & -1
\end{array}\right]
$$

Evidently, we have

$$
M_{2} \times\left[\begin{array}{c}
(0,0) \\
(0,1) \\
(-1,0) \\
(-1,1)
\end{array}\right] \equiv-\left[\begin{array}{c}
(0,0) \\
(0,1) \\
(-1,0) \\
(-1,1)
\end{array}\right]=\left[\begin{array}{c}
(0,0) \\
(-1,0) \\
(0,1) \\
(-1,1)
\end{array}\right]
$$

Certain B-NOT and B-NEG equivalence could be technically interesting in quantum gate design such as

$$
\begin{aligned}
& \neg\left[\begin{array}{c}
(-1,0) \\
(0,1)
\end{array}\right] \equiv-\left[\begin{array}{c}
(-1,0) \\
(0,1)
\end{array}\right]=\left[\begin{array}{c}
(0,1) \\
(-1,0)
\end{array}\right] ; \\
& -\left[\begin{array}{c}
(0,0) \\
(-1,1)
\end{array}\right]=\left[\begin{array}{c}
(0,0) \\
(-1,1)
\end{array}\right]
\end{aligned}
$$

Similarly, we can define other bipolar gates such as B- $\otimes$ and B- $\oplus$ gates. It can be shown that Toffoli gate can be extended to bipolar Toffoli gate for simulating bipolar NAND gate. This will be reported in another paper.

\subsection{Quantum Mechanical Implementations}

Physical implementation of BDL or BQLA can be in different ways such as using quantum dots, atoms, trapped ions or superconductors. A bipolar qubit register [12, Ch.7] is shown in Figure 6(a), which consists of two Bloch spheres, one holds a negative quantum dot and another holds a positive one. When both dots are inside the rectangle box it may register a $|(-1,+1)\rangle$ state; if both are outside the box, it may register a $|(0,0)\rangle$ bipolar state; if the negative dot is inside but the positive one is outside, it may register a $|(-1,0)\rangle$ state; otherwise, it may register a $|(0,+1)\rangle$ state. When probability coefficients are taken into consideration, the box creates a macroscopically coherent bipolar superposition, of the form $c 00|(0,0)\rangle+c 01|(0,1)\rangle+c 10|(-1,0)\rangle+c 11|(-1,+1)\rangle$. The bipolar quantum register seems to present a white-box resolution to Schrödinger's cat paradox.

The bipolar superposition is fundamentally different from that of a traditional 2-qubit system in the form of $c 00|00\rangle+c 01|01\rangle+c 10|10\rangle+c 11|11\rangle$. While the traditional basis states are binary bit patterns without quantum causality and basis state entanglement, the bipolar basis states are logical values in BDL that form a bipolarcausal sets and lead to basis state quantum entanglement formally defined in the form of BUMP. (Note: Without losing generality we limit the scope of this work on basis state quantum causality and entanglement). 


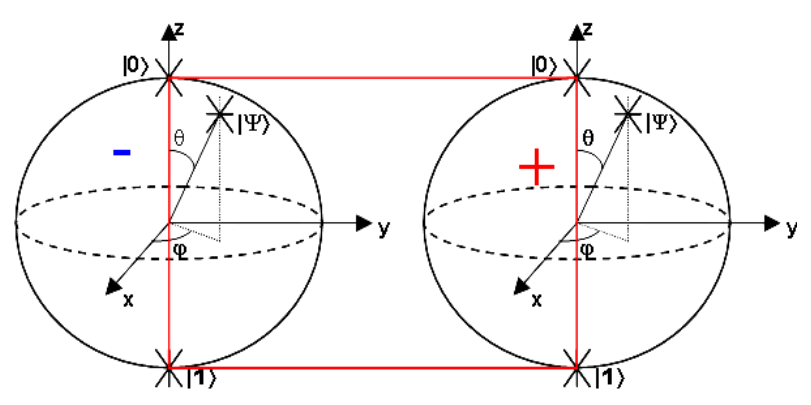

(a)

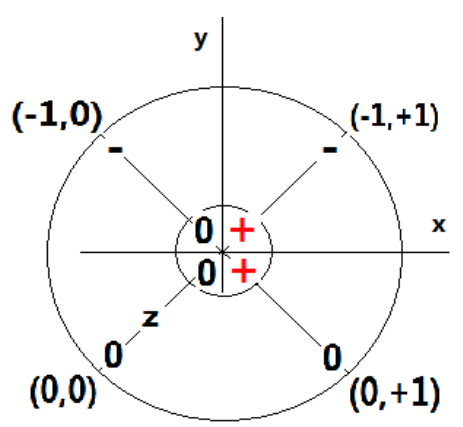

(b)

Figure 6. (a) Bipolar Qubit; (b) Bipolar Bloch sphere of a 2-electron system.

Alternatively, a 2-electron model (Figure 6(b)) can be used to illustrate equilibrium or symmetry with modularity. The entanglement of two electron systems has been reported in the literature [16]. But, to the author's knowledge, besides BDL no other causal logic is reported for such a system. An immediate question is measurability.

Three reasonable assumptions can be made:

(1) $(-1,+1)$ is stronger than $(-1,0)$ in spin energy;

(2) $(0,0)$ remains symmetrical to $(-1,+1)$;

(3) $(0,+1)$ remains symmetrical to $(-1,0)$.

It is clear that B-NOT gate can flip the quantum states in Figure 6(b) to their symmetrical ones in Figure 7(a). Such a flip is reversible both physically and logically. Logically, we have $E \equiv \neg(\neg E)$. Similarly, B-NEG gate is also reversible with $E \equiv-(-E)$ as shown in Figure 7(b).

\subsection{Generic Bipolar Quantum Entanglement}

Mainstream quantum computing inherits some binary features from classical digital computing. This heritage has never been challenged. Despite repeated verification of faster than light observations [17], quantum causality and entanglement finds no logical interpretation. Bipolar quantum causality and entanglement present a major deviation from the binary heritage and it may hold the key for scalable modular quantum logic gate design.

It is interesting to note that two entangled single electron systems, no matter how far away they are separated,

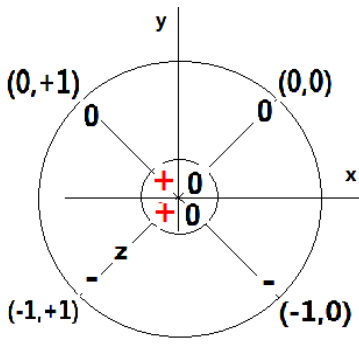

(a)

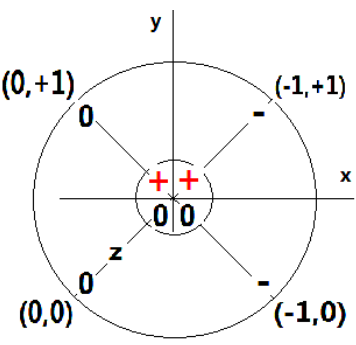

(b)
Figure 7. (a) Result by applying B-NOT gate on Figure 6(b); (b) Result by applying B-NEG gate on Figure 6(b).

should satisfy bipolar quantum causality as defined by BUMP (Table 3). BUMP in the form $(\phi \Leftrightarrow \varphi) \Rightarrow$ $[(\phi * \psi) \Leftrightarrow(\varphi * \psi)]$ simply states: If bipolar logical variables $\phi$ and $\varphi$ are bipolar equivalent (or entangled) in the background independent YinYang bipolar geometry (Figure 4), any bipolar interaction $\phi * \psi$ implies the bipolar interaction $\varphi * \psi$ or vice versa. Thus, BUMP defines a bipolar causal relation that provides the first equilibrium-based logical interpretation for the mysterious quantum correlation.

It is posited $[12,14,15]$ that:

Postulate 1. Bipolar quantum entanglement is the most fundamental entanglement.

Postulate 2. YinYang bipolarity is the most fundamental property of the universe.

The two postulates are actually logically provable axioms. For postulate 1 if a bipolar value characterizes the energy superposition of gravitational and quantum action-reaction, an atom would be a set of bipolar elements (Figure 6). As the total must be equal to the sum, without bipolar quantum entanglement there would be no atom level quantum entanglement. Postulate 2 follows postulate 1 .

Another proof comes from established observations in quantum mechanics. It is well-known that pairs of particles can be generated so that they are in some specific quantum states. For instance, a pair of these particles may be generated having a two-state spin: when one spin is up the other must be down. This type of entangled pair with opposite spin, is known as the spin anti-correlated case. This kind of entanglement is central in quantum teleportation and cryptography but so far it has not been described in logical form. With BUMP in BDL, the entanglement can be characterized as

$$
(0,+1) \Leftrightarrow-(-1,0) \text { or }(0,+1) \Leftrightarrow \neg(-1,0) \text {. }
$$

Generalization of Bipolar Quantum Entanglement. To further extend BDL for quantum causality, we use $\Omega$ as a generalized symbol to include spin anti-correlated entanglement in addition to $\Leftrightarrow$. With this generalization, all observed generic cases can be characterized as, $\forall(x, y),(u, v) \in B_{1}$, either 


$$
(x, y) \Omega(u, v) ;(x, y) \Omega-(x, y) ; \text { or }(x, y) \Omega \neg(x, y) .
$$

Thus, BUMP has led to generic level quantum entanglement in a background independent bipolar geometry. A bipolar qubit register (Figure 6(a)) can characterize a bipolar variable in BUMP for generic bipolar quantum entanglement. Evidently, B-Neg and B-Not gates may play an important role in quantum entanglement.

\subsection{Composite Quantum Entanglement}

Generic entanglement is logically strict. However, in the generic case variables in BUMP has to be fundamental subatomic particles, antiparticles, or a pair of them that doesn't account for atom level entanglement with many such pairs of subatomic particles.

While Figure 6(b) is a simple illustration to the two postulates, Figure 8 gives a more obvious illustration where both matter and antimatter atoms can be characterized as a composite set of bipolar elements and unified with Equation (15) [14].

From a computational perspective, bipolar causality can be characterized by measuring one entangled atom and then to predict the other far away like in quantum teleportation. For instance, if two entangled and then separated atoms have the states

$$
\left[\begin{array}{c}
(-1,0) \\
(0,1) \\
(0,0) \\
(-1,1)
\end{array}\right] \Omega\left[\begin{array}{c}
(-1,0) \\
(0,1) \\
(0,0) \\
(-1,1)
\end{array}\right]
$$

By applying the B-NEG operation to the first atom, the state of the $2^{\text {nd }}$ one can be predicted using the following equivalence within the background independent bipolar geometry:

$$
-\left[\begin{array}{c}
(-1,0) \\
(0,1) \\
(0,0) \\
(-1,1)
\end{array}\right] \Omega-\left[\begin{array}{c}
(-1,0) \\
(0,1) \\
(0,0) \\
(-1,1)
\end{array}\right]
$$

The above example is intuitive as both sides are in the same order. Interestingly, we may have the following entanglement:

$$
\left[\begin{array}{c}
(-1,0) \\
(0,1) \\
(0,0) \\
(-1,1)
\end{array}\right] \Omega-\left[\begin{array}{c}
(-1,0) \\
(0,1) \\
(0,0) \\
(-1,1)
\end{array}\right]
$$

This is a rather counterintuitive but a possible spin anti-correlated case. The counterintuitive nature is due to the background independent property of YinYang bipolar geometry. However, the entanglement (if possible) does not violate any equilibrium, symmetry and reversibility principles. In quantum lattice form it is equivalent to $\left(B_{1}\right) \Omega\left(-B_{1}\right)$ (see Figure 9).

Similarly, we may have the following complementary entanglement:

$$
\left[\begin{array}{c}
(-1,0) \\
(0,1) \\
(0,0) \\
(-1,1)
\end{array}\right] \Omega \neg\left[\begin{array}{c}
(-1,0) \\
(0,1) \\
(0,0) \\
(-1,1)
\end{array}\right]
$$

which can be equivalently described in quantum lattice form $\left(B_{1}\right) \Omega\left(\neg B_{1}\right)$ in Figure 10. From Figure 10, it is clear that the composite entanglement (if possible) does not violate any equilibrium, symmetry and reversibility principles.

Furthermore, assuming reversible quantum entanglement or teleportation we would have faster than light information transmission such as $\left(-B_{1}\right) \Omega\left(--B_{1}\right)$ and $\left(\neg B_{1}\right) \Omega\left(\neg \neg B_{1}\right)$ :
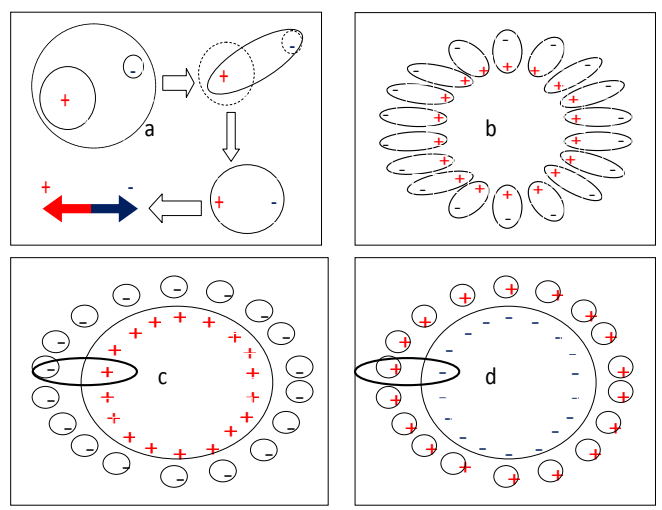

Figure 8. (a) Bipolar representation of hydrogen atom; (b) YinYang n-elements; (c) Matter atom; (d) Antimatter atom.

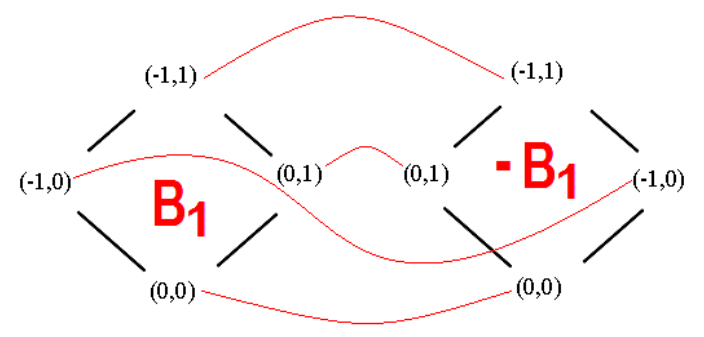

Figure 9. Quantum lattice description $\left(B_{1}\right) \Omega\left(-B_{1}\right)$.

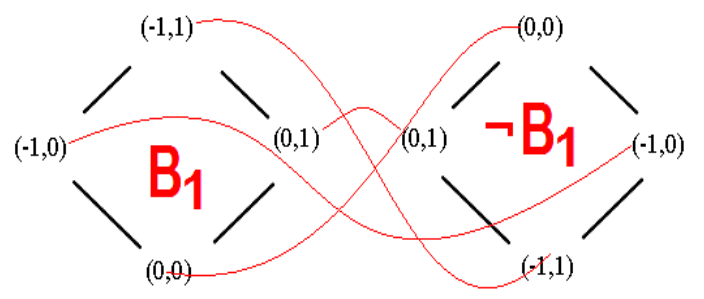

Figure 10. Quantum lattice description $\left(B_{1}\right) \Omega\left(\neg B_{1}\right)$. 


$$
-\left[\begin{array}{c}
(-1,0) \\
(0,1) \\
(0,0) \\
(-1,1)
\end{array}\right] \Omega--\left[\begin{array}{c}
(-1,0) \\
(0,1) \\
(0,0) \\
(-1,1)
\end{array}\right] \text { and } \neg\left[\begin{array}{c}
(-1,0) \\
(0,1) \\
(0,0) \\
(-1,1)
\end{array}\right] \Omega \neg \neg\left[\begin{array}{c}
(-1,0) \\
(0,1) \\
(0,0) \\
(-1,1)
\end{array}\right]
$$

\subsection{Reversibility}

Bipolar quantum entanglement should be classified as background independent basis state entanglement in YinYang bipolar geometry where quadrant is irrelevant (Figure 4). This property makes logical reversibility $[18,19]$ possible for BDL.

Theorem 1 (Reversibility Theorem): Bipolar quantum logic gate design can achieve logical reversibility-a step toward bipolar universal quantum computing.

Proof. It follows from $(A) B D L$ subsumes Boolean logic [12, Ch.3]; (B) B-NOT and B-NEG gates are both reversible; and $(C)$ the following logical equivalence observations hold (see proof in [12, Ch.3]:

$B-A N D(\&)$ gate is bipolar linearly equivalent to Boolean AND gate.

B-OR $(\oplus)$ gate is bipolar linearly equivalent to Boolean OR gate.

B-TENSOR $(\otimes)$ gate can be decomposed to the following cases:

(1) $(x, y) \otimes(0,0) \equiv(0,0)$, equivalent to NULL gate;

(2) $(x, y) \otimes(0,1) \equiv(x, y)$, equivalent to IDENTITY gate;

(3) $(x, y) \otimes(-1,0) \equiv-(x, y)$, equivalent to $B-N E G$ gate;

(4)

$(x, y) \otimes(-1,1) \equiv[(x, y) \otimes(-1,0)] \oplus[(x, y) \otimes(0,1)]$, that is equivalent to the $B$-OR of a $B-N E G$ operation.

From the four cases we see that case (1) is the NULL law; case (2) is the identity law; case (4) is a composition of case (2) and (3); the essential case is number (3)-the $B-N E G$ gate. Since the other gates $\&^{-}, \oplus^{-}$, and $\otimes^{-}$are the $B-N E G$ of $\&, \oplus$ and $\otimes$, respectively, and since existing (unipolar or 2-dimensional) quantum logic gates have achieved reversibility, it is clear that bipolar logical reversibility is achievable based on $B D L$.

\section{Quantum Cellular Combinatorics}

\subsection{An Equilibrium/Non-Equilibrium Approach}

Combinatorics is a branch of mathematics concerning the study of finite or countable discrete structures. Aspects of combinatorics include counting the structures of a given kind and size, deciding when certain criteria can be met, and constructing and analyzing objects meeting the criteria, finding "largest", "smallest", or "optimal" objects, and studying combinatorial structures arising in an algebraic context, or applying algebraic techniques to com- binatorial problems (algebraic combinatorics).

Combinatorial problems arise in mathematics with many applications. One of the oldest and most accessible parts of combinatorics is graph theory, which also has numerous natural connections to other areas. Combinatorics is used frequently in computer science to obtain formulas and estimates in the analysis of algorithms.

BDL and BQLA provide a unique logical and mathematical basis for bipolar quantum combinatorics. The new approach is equilibrium-based on the negative or positive energies [20] or the Yin and Yang of nature [14,15,21-29]. This focus is expected to foster ubiquitous quantum computing and quantum intelligence in both social and natural science.

\subsection{Combinatorial YinYang-1-Element Graph}

Figure 11 shows the graph of a YinYang-1-element as the most basic structure of QCC. This element seems to be rather simple. But a closer examination reveals its quintessential role as the smallest and, at the same time, the largest structure in the new type of combinatorics for quantum cellular computing.

First, we consider it as a smallest agent with equilibrium or non-equilibrium. In this case, it can be used as a model for a particle-antiparticle pair variable $E_{1}=$ $\left(e^{-}, e^{+}\right)$or an energy input-output variable. For instance, if $\left(e^{-}, e^{+}\right)=(-1,0)$ it can represent an electron or non-equilibrium; if $\left(e^{-}, e^{+}\right)=(0,+1)$ it can represent a positron or another non-equilibrium; if $\left(e^{-}, e^{+}\right)=$ $(-1,+1)$ it can represent an electron-positron pair or an energy equilibrium; if $\left(e^{-}, e^{+}\right)=(0,0)$ it can represent an annihilation of the pair or eternal equilibrium.

Interestingly, the reflexive link can also be bipolar to add dynamic change or mutation to the agent. Different from unipolar reflexivity, in the bipolar case we may have positive reflexivity $(0,+|x|)$, negative reflexivity $(-|x|, 0)$, or bipolar reflexivity $(-|x|,+|y|)$ characterized with three different colors, respectively. For instance, if the reflexive link weight is $(-1,0)$, when $\mathrm{n}$ is odd we have $(-1,0) \otimes(-1,0) \otimes \cdots \otimes(-1,0)=(-1,0)^{n}=(-1,0)$ and when $\mathrm{n}$ is even we have $(-1,0)^{n}=(0,+1)$. This property seems rather bizarre but it can represent the most fundamental natural or biological oscillation processes in microscopic as well as macroscopic worlds. For instances, a subatomic particle can change polarity three trillion times per second between matter to antimatter [30]; some genetic agent exhibits YinYang bipolar repression-activition $(-,+)$ abilities in gene expression regulation [22].

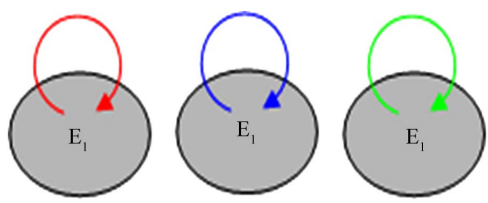

Figure 11. Yin yang-1-element. 
Secondly, we consider YinYang-1-element as the largest equilibrium or non-equilibrium. Evidently, our universe can switch from big bang $(0,+1)$ state to a black hole state $(-1,0)$. This can be characterized as $(0,+1) \otimes(-1,0)=(-1,0)$. Interestingly, it may also switch from a black hole state to a big bang state which can be characterized as $(-1,0) \otimes(-1,0)=(0,+1)$. In this case, we have a cyclic process model $(-1,0)^{n}$ for our universe.

If we assume a negative reflexive link and an adaptive bipolar element, we could have

$$
\begin{aligned}
& (-1,0) \oplus(-1,0)^{2} \\
& =(-1,0) \oplus[(-1,0) \otimes(-1,0)] \\
& =(-1,0) \oplus(0,+1)=(-1,+1)
\end{aligned}
$$

which shows a self-adaptation of $(-1,0)$ to bipolar equilibrium $(-1,+1)$ for an agent, small or large [12]. The key is self adaptivity characterized by $\oplus$ and $\otimes$ in combination with memory ability.

Of course, if reflexivity is defined as $\times$ instead of $\otimes$ and the reflexive link weight is bipolar balanced, $\forall(x, y),(u, v) \in B_{\infty}$ and $|u|+v=1.0$, following Equation (13), we have $(x, y) \times(u, v) \equiv(x v+y u, x u+y v)$ and, in addition, we must have

$$
|x|+y \equiv(|x v+y u|)+(x u+y v) .
$$

Remarkably, Equation (17) defines the law of global energy equilibrium regardless of local bipolar balance or imbalance. This exhibits the significance of the bipolar generalization. Without bipolarity, causality, quantum entanglement and energy equilibrium would be impossible.

Thirdly, we consider YinYang-1-element as a medium-sized equilibrium or non-equilibrium. This may sound impossible. But, evidently, a person's mind can be in depression $(-1,0)$, mania $(0,+1)$, equilibrium $(-1$, $+1)$, eternal equilibrium $(0,0)$ or between $(-0.6,+0.7)$.

Actually, all human beings have to be in either mental equilibrium or non-equilibrium or between. To certain extent, we are all mentally bipolar, either in equilibrium or disorder or between because no one's mind can escape equilibrium or non-equilibrium and bipolar equilibrium/ non-equilibrium is most fundamental. In this case

$(-1,0) \oplus(-1,0)^{2}$ can characterize the mental adaptation from depression to equilibrium $[31,32]$.

\subsection{Combinatorial YinYang-2-Element Graph}

Figure 12 shows the structures of a YinYang-2-element graph as the $2^{\text {nd }}$ most basic structure of QCC for bipolar interaction. These structures added two more bipolar links between the two bipolar elements in equilibrium or non-equilibrium (green: harmonic; red: positive; blue: negative).
A link weight can be any $(x, y)$ in $B_{\infty}$. For instance, $(0,0)$ shows no interaction; $(-1,0)$ shows conflict or inhibition to each other; $(0,+1)$ shows coalition or excitation to each other; $(-1,+1)$ shows harmonic interaction. As a basic combinatorial graph for equilibrium or non-equilibrium bipolar interaction, YinYang-2-element is critical in characterizing bipolar quantum entanglement for building larger combinatorial networks.

\subsection{Combinatorial YinYang-N-Element Graph}

Figure 13 shows YinYang-3- and YinYang-4-element graphs. Figure 14 shows a YinYang-5-element graph. Figure 15 shows a YinYang-n-element graph. The 3-

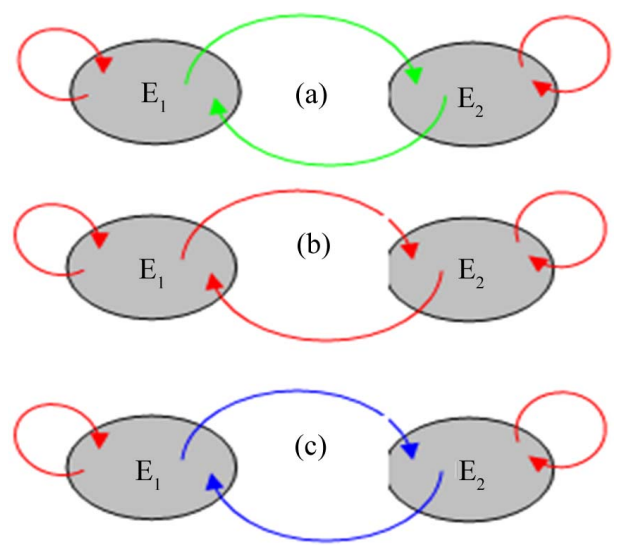

Figure 12. Yin yang-2-element.
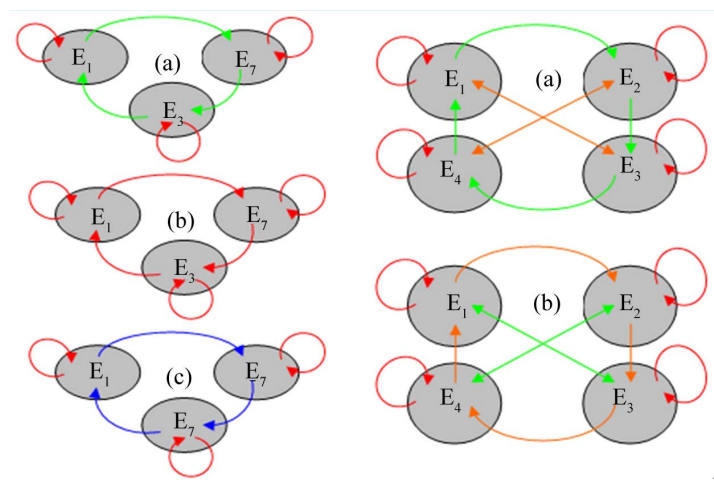

Figure 13. YinYang-3-element and YinYang-4-element.

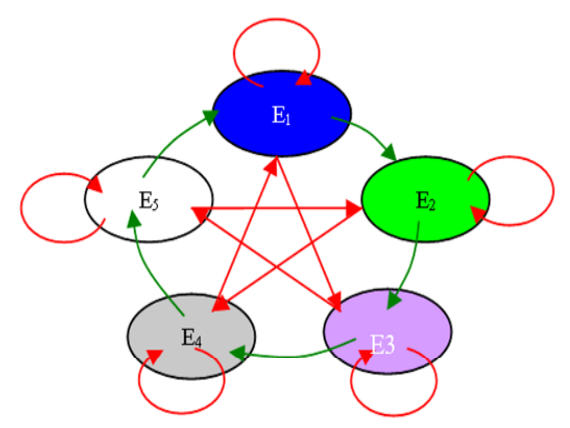

Figure 14. YinYang-5-element. 


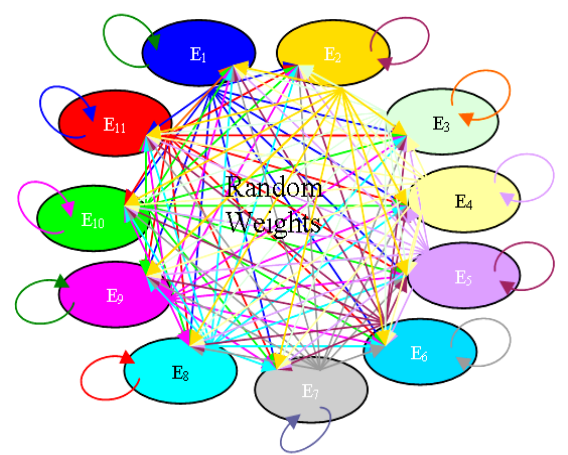

Figure 15. Yin Yang-n-element.

and 4-element graphs both exhibit some intrinsic properties that deserve further investigation. The YinYang-5element structure is historically prominent in Chinese cosmology and traditional Chinese medicine. YinYangn-element graph is general and central in QCC.

As a causal set or relation, YinYang-n-element graph leads to the concept of bipolar transitive closure. The bipolar transitive closure of a bipolar relation $R$ is the smallest transitive bipolar relation containing $R[9,12]$, denoted by $\Re$ and

$$
\mathfrak{R}=R^{1} \oplus R^{2} \oplus R^{3} \oplus \cdots
$$

It is found [9,12] that, let $X=\left\{x_{1}, x_{2}, \cdots, x_{n}\right\}$ be a finite bipolar set, the bipolar transitive closure $\mathfrak{R}$ of $R$ in $X$ exists, is unique, and

$$
\Re=R^{1} \oplus R^{2} \oplus R^{3} \oplus \cdots \oplus R^{2 n} .
$$

The concept of bipolar transitive closure has led to the generalizations of equivalence relations to bipolar equilibrium relations and fuzzy similarity relations to bipolar fuzzy or quasi-equilibrium relations [9,33]. Based Equation (19), algorithms have been devised for bipolar clustering from equilibrium relations. While equivalence relations lead to hard partitions of equivalence sets; equilibrium relations lead to the partitions of coalition sets, conflict sets, and harmonic sets $[9,33]$.

From a dynamic equilibrium perspective we have:

Law 1. Elementary Energy Equilibrium Law.

$$
\forall(x, y) \in B_{\infty}=[-\infty, 0] \times[0,+\infty] \text { and } \forall(u, v) \in B_{F}=
$$
$[-1,0] \times[0,1]$, let $|\varepsilon|(x, y)=|x|+y$, we have

$$
\begin{aligned}
& {[|\varepsilon|(u, v) \equiv 1.0] \Rightarrow[|\varepsilon|((x, y) \times(u, v)) \equiv|\varepsilon|(x, y)] ;} \\
& {[|\varepsilon|(u, v)<1.0] \Rightarrow[|\varepsilon|((x, y) \times(u, v))<|\varepsilon|(x, y)] ;} \\
& {[|\varepsilon|(u, v)>1.0] \Rightarrow[|\varepsilon|((x, y) \times(u, v))>|\varepsilon|(x, y)] .}
\end{aligned}
$$

Equilibrium/Non-Equilibrium System. A bipolar dynamic cellular system $S$ is said an equilibrium system if the system's total energy $|\varepsilon| S$ remains in an equilibrium state or $\mathrm{d}(|\varepsilon| S) / \mathrm{d} t=0$ without external disturbance. Otherwise it is said a non-equilibrium system. A non-equilibrium system is said a strengthening system if $\mathrm{d}(|\varepsilon| S) / \mathrm{d} t>0$; it is said a weakening system if $\mathrm{d}(|\varepsilon| S) / \mathrm{d} t<0$.

Law 2. Energy Transfer Equilibrium Law. Following Equation (16), we have

1) As long as $M$ is bipolar energy conservational, $V=M$ $x E$ remains a global equilibrium system regardless of local equilibrium or non-equilibrium for its elements or $|\varepsilon|(V)=|\varepsilon|(M \times E) \equiv|\varepsilon|(E)$.

2) As long as the absolute energy total of every row and column of matrix $\mathrm{M}$ is greater than 1.0, $\forall V, M, E$, $V=M \times E$ remains a globally strengthening system or $|\varepsilon|(V)=|\varepsilon|(M \times E)>|\varepsilon|(E)$, regardless of local weakening or strengthening for its different elements.

3) As long as the absolute energy total of every row and column of matrix $M$ is smaller than 1.0, $\forall V, M, E, V$ $=M \times E$ remains a globally weakening system or $|\varepsilon|(V)$ $=|\varepsilon|(M \times E)<|\varepsilon|(E)$, regardless of local weakening or strengthening for its different elements

Based on the above two laws, the laws of energy symmetry, broken symmetry, and oscillation as well as the laws of cellular integration have been formulated. It is shown in $[12,14]$ that these laws can be used for the unification of particle and wave as well as matter and antimatter.

\section{Analysis and Discussion}

Five years after publishing their EPR paradox paper, Einstein asserted [34], "For the time being we have to admit that we do not possess any general theoretical basis for physics which can be regarded as its logical foundation." The bipolar approach to quantum mechanics and quantum computing can be deemed a logical approach to physics, quantum mechanics and quantum computing. It exhibits a number of unique properties.

Equilibrium-Based Generalization of Truth. BDL is an equilibrium-based bipolar dynamic generalization of Boolean logic following the $2^{\text {nd }}$ law of thermodynamics. It can be recovered to Boolean logic and therefore is digitally computational [12]. On the other hand, since all truths including quantum and non-quantum truths must exist in certain equilibrium or non-equilibrium, BDL provides a logical basis for ubiquitous quantum computing and intelligence.

Causal Logic. BDL provides logically definable causality for the first time ever in the history through BUMP [12].

Bipolar Quantum Entanglement. Logically definable causality may lead to reversible quantum entanglement which may, in turn, lead to reversible quantum teleportation for faster than light information transmission $[12,17]$.

Quantum-Digital Compatibility and Bitwise Encryption. An OR operation on the absolute values of the 
two poles will recover BDL to Boolean logic [12]. This provides a logical basis for quantum-digital compatibility and hybrid computability. On the other hand, BDL and BQLA are equilibrium-based logical but truth-based illogical. This property can be used for bipolar coding and bitwise quantum encryption [12] that may lead to quantum cryptography immune to factorization-based algorithms [35]. Furthermore, bipolar coding can be a pure software scheme independent of a quantum computer.

Fault Tolerance. The bipolar decimal generalization of quantum gate matrices may find applications in fault tolerance analysis in quantum computation.

Universality. With the theoretical basis of bipolar reversibility, an immediate research topic is to extend Toffoli gate and Hadamaard gate to bipolar quantum logic gates for phase shift, superposition and universal quantum computing. It is expected an achievable task.

Modularity. Bipolar quantum logic gates provide an important level of abstraction that may enhance modularity and scalability in large-scale quantum circuit design. While this work is focused on the logical aspects, some physical aspects are left for further investigation.

Quantum Intelligence. Quantum cellular combinatorics has been made possible by this work. It is expected to aid quantum logic gate design and quantum algorithm analysis. It is also expected to foster quantum intelligence - a unification of machine or artificial intelligence with biological and social intelligence.

Logical Unification. Following Aristotle, Einstein, Bohr and Bell, quantum mechanics and computing have been shaped as unipolar paradigms based on bivalent truth [3-5,36]. This work has attempted to reshape the field with a holistic bipolar equilibrium-based approach. Logically definable quantum causality and entanglement has provided a logical extension to Bohr's particle-wave complementarity, Bohm's wave function and Bell's theorem. Dramatically, the extension can also be deemed a possible resolution, rather than falsification, to the EPR paradox. More specifically, given two entangled bipolar variables, local bipolar equilibrium or non-equilibrium can be deemed the hidden parameter or local reality as suggested by the EPR argument. On the other hand, the locality of a dynamic equilibrium, unlike a truth object, has to be part of a global dynamic equilibrium that can lead to quantum non-locality or "spooky action in a distance." This may also leads to spacetime emergence.

Possible Physical Unification. It is posited [12,14] that all action-reaction objects in the universe may be quantum entangled through gravitational and quantum mechanical forces. Since the effects of acceleration are indistinguishable from that of gravitation per general relativity, should gravitation travels at the same speed of quantum entanglement $[12,14,17]$, action-reaction (-F, $+F)$ in classical mechanics may involve a usually negli- gible time delay from $+\mathrm{F}$ to $-\mathrm{F}$ at the speed of quantum entanglement. In that case, Newton mechanics, general relativity and quantum mechanics would all be unified under bipolar quantum entanglement.

Postuate 3. The universe is a bipolar quantum entanglement.

Postuate 4. Any world, physical, biological, social or mental, can be modeled in some way as a bipolar quantum logic gate or a graph of bipolar quantum cellular combinatorics.

Pervasiveness and Falsifiability. Falsifiability is a key criterion of any scientific theory. The century old search for monopole has so far failed. Without monopole, string theory is deemed not falsifiable and criticized "Not Even Wrong" [37]. But dipoles, particle-antiparticle pairs, action-reaction objects, repression-activation, negative-positive energies or the Yin and Yang of nature are observable reality. Furthermore, spin anticorrelated quantum entanglement with faster than light observation [17] as well as the ubiquitous nature of bipolar coexistence seems to support bipolar quantum entanglement. After all, generic bipolar quantum entanglement can be defined as a logical formula by BUMP. Its logical falsifiability is self-evident regardless of its physical existence or non-existence.

\section{Conclusions}

Bipolar quantum logic gates and quantum cellular combinatorics have been presented with a logical interpretation to quantum entanglement. A number of unique properties of bipolar quantum logic gates have been revealed. It has been shown that, with logically definable causality, BDL and BQLA lead to generic and composite levels of bipolar quantum entanglement. Logical equivalence to established theories has been identified with well-observed quantum phenomena. A reversibility theorem has been presented as a first step toward universal bipolar quantum computing.

It has been shown that a bipolar matrix can be either a modular generalization of a quantum logic gate matrix or a bio-cellular connectivity matrix. Based on this observation, a modular and scalable graph theory of quantum cellular combinatorics has been proposed for quantum intelligence.

It has been contented that logically definable quantum causality and entanglement provide an equilibrium-based logical extension to Bohr's particle-wave complementarity principle, Bohm's wave function and Bell's theorem. In the meantime, it has also been suggested that the extension is not necessarily a falsification but a unification of quantum non-locality and local realism as suggested by the EPR thought experiment.

Quantum entanglement discussed in this work is lim- 
ited in the scope of basis states. This limitation provides a focus on some most fundamental properties of bipolar quantum logic gates and quantum cellular combinatorics. The limitation can be lifted with bipolar quantum superposition that is left for future research effort.

Finally, it should be remarked that the word "realism" bears with two fundamentally different philosophical connotations. This disparity is originated from the historical student-teacher dispute between Aristotle and his teacher Plato. Platonic realism is usually used to refer to the idea of universals or abstract objects. As universals were considered by Plato to be ideal forms, this stance is confusingly also called Platonic idealism. In Aristotle's view, however, universals exist only where they are instantiated; they exist only in things, never apart from things. Scientific local realism has taken Aristotle's side for most of the times. Now the phenomenon of quantum entanglement is changing the tide. Many be- lieve that local realism is deemed unrealistic in the quan- tum world. The equilibrium-based approach to quantum entanglement seems to present a logical unification of both sides. In this unification, Aristotle's local realism (as advocated in the EPR argument) is accounted for by truth-object(s) hosted in local equilibrium or nonequilibrium and Platonic global realism (as advocated by Bohr's quantum non-locality) is accounted for with global equilibrium or non-equilibrium. Since nothing can escape from equilibrium or non-equilibrium, BDL and BQLA have made the unification possible.

\section{Acknowledgements}

The author acknowledges Lotfi Zadeh for his recognition [38] of the author's work that supports logically definable causality and the anonymous reviewers for their valuable suggestions that helped the revision and finalization of this work.

\section{REFERENCES}

[1] S. Blackburn, "Hume and Thick Connexions," Philosophy and Phenomenological Research, Vol. 50, 1990, pp. 237-250. doi: $10.2307 / 2108041$

[2] L. A. Zadeh, "Causality Is Undefinable-Toward a Theory of Hierarchical Definability," Proceedings of FUZZIEEE, Melbourne, 2-5 December 2001, pp. 67-68

[3] J. S. Bell, "On the Einstein-Podolsky-Rosen Paradox," Physics, Vol. 1, No. 3, 1964, pp. 195-200.

[4] A. Einstein, B. Podolsky and N. Rosen, "Can QuantumMechanical Description of Physical Reality Be Considered Complete? Physical Review, Vol. 47, No. 10, 1935, pp. 777-780. doi:10.1103/PhysRev.47.777

[5] N. Bohr, "On The Notions of Causality and Complementarity," Dialectica, Vol. 2, No. 3-4, 1948, pp. 312-319. doi:10.1111/j.1746-8361.1948.tb00703.x
[6] K. Hess and W. Philipp, "A Possible Loophole in the Theorem of Bell," Proceedings of the National Academy of Sciences of USA, Vol. 98, No. 25, 2001, pp. 1422414227. doi: $10.1073 /$ pnas.251524998

[7] D. Bohm, "Causality and Chance in Modern Physics," University of Pennsylvania Press, Philadelphia, 1980.

[8] M. Kumar, "Quantum: Einstein, Bohr, and the Great Debate about the Nature of Reality," Norton \& Company, New York/London, 2010.

[9] W.-R. Zhang, "Equilibrium Relations and Bipolar Cognitive Mapping for Online Analytical Processing with Applications in International Relations and Strategic Decision Support," IEEE Transactions on SMC, Part B, Vol. 33. No. 2, 2003, pp. 295-307.

[10] W -R. Zhang and L. Zhang, "YinYang Bipolar Logic and Bipolar Fuzzy Logic," Information Sciences, Vol. 165, No. 3-4, 2004, pp. 265-287.

doi:10.1016/j.ins.2003.05.010

[11] W.-R. Zhang, "YinYang Bipolar Lattices and L-Sets for Bipolar Knowledge Fusion, Visualization, and Decision," International Journal of Information Technology and Decision Making, Vol. 4, No. 4, 2005, pp. 621-645 doi:10.1142/S0219622005001763

[12] W.-R. Zhang, "YinYang Bipolar Relativity: A Unifying Theory of Nature, Agents and Causality with Applications in Quantum Computing, Cognitive Informatics and Life Sciences," IGI Global, Hershey, 2011.

[13] L. Bombelli, J. Lee, D. Meyer and R. D. Sorkin, "Spacetime as a Causal Set," Physical Review Letters, Vol. 59, No. 5, 1987, pp. 521-524. doi:10.1103/PhysRevLett.59.521

[14] W.-R. Zhang, "YinYang Bipolar Atom: An Eastern Road toward Quantum Gravity," Journal of Modern Physics, Vol. 3, 2012, pp. 1261-1271. doi:10.4236/jmp.2012.329163

[15] W.-R. Zhang, "Beyond Spacetime Geometry: The Death of Philosophy and Its Reincarnation," Journal of Modern Physic, Vol. 3, 2012, pp. 1272-1284. doi:10.4236/jmp.2012.329164

[16] R. J. Yañez, A. R. Plastino and J. S. Dehesa, "Quantum Entanglement in a Soluble Two-Electron Model Atom," The European Physical Journal D, Vol. 56, No. 1, 2010, pp. 141-150. doi:10.1140/epjd/e2009-00270-x

[17] D. Salart, A. Baas, C. Branciard, N. Gisin and Z. Hugo, "Testing the Speed of 'Spooky Action at a Distance'," Nature, Vol. 454, 2008, pp. 861-864. doi:10.1038/nature07121

[18] C. H. Bennett, "Logical Reversibility of Computation," IBM Journal of Research and Development, Vol. 17, 1973, pp. 525-532. doi:10.1147/rd.176.0525

[19] T. Toffoli, "Reversible Computing," Tech. Memo MIT /LCS/TM-151, MIT Lab. for Com. Sci., Boston, 1980.

[20] S. Hawking and L. Mlodinow, "The Grand Design," Random House Digital, Inc., New York, 2010.

[21] J. Gore and A. van Oudenaarden, "Synthetic Biology: The Yin and Yang of Nature," Nature, Vol. 457, No. 7227, 2009, pp. 271-272. doi:10.1038/457271a 
[22] Y. Shi, E. Seto, L.-S. Chang and T. Shenk, "Transcriptional Repression by YY1, a Human GLI-Kruppel-Related Protein, and Relief of Repression by Adenovirus E1A Protein," Cell, Vol. 67, No. 2, 1991, pp. 377-388. doi:10.1016/0092-8674(91)90189-6

[23] B. M. Jacobsen and D. G. Skalnik,"YY1 Binds Five cis-Elements and Trans-Activates the Myeloid Cell-Restricted gp91phox Promoter," The Journal of Biological Chemistry, Vol. 274, 1999, pp. 29984-29993. doi:10.1074/jbc.274.42.29984

[24] H. Liu, M. Schmidt-Supprian, Y. Shi, E. Hobeika, N. Barteneva, H. Jumaa, R. Pelanda, M. Reth, J. Skok, K. Rajewsky and Y. Shi, "Yin Yang 1 Is a Critical Regulator of B-Cell Development," Genes \& Development, Vol. 21, 2007, pp. 1179-1189. doi:10.1101/gad.1529307

[25] L. Palko, H. W. Bass, M. J. Beyrouthy and M. M. Hurt, "The Yin Yang-1 (YY1) Protein Undergoes a DNA-Replication-Associated Switch in Localization from the Cytoplasm to the Nucleus at the Onset of S Phase," Journal of Cell Science, Vol. 117, 2004, pp. 465-476. doi: $10.1242 /$ jes. 00870

[26] S. Vasudevan, Y. Tong and J. A. Steitz, "Switching from Repression to Activation: MicroRNAs Can Up-Regulate Translation," Science, Vol. 318, No. 5858, 2007, pp. 19311934. doi:10.1126/science. 1149460

[27] W.-R, Zhang, H. J. Zhang, Y. Shi and S. S. Chen, "Bipolar Linear Algebra and YinYang-N-Element Cellular Networks for Equilibrium-Based Biosystem Simulation and Regulation," Journal of Biological Systems, Vol. 17, No. 4, 2009, pp. 547-576. doi:10.1142/S0218339009002958

[28] W.-R. Zhang, S. Chen and J. C. Bezdek, "A Generic System for Cognitive Map Development and Decision Analysis," IEEE Transactions on Systems, Man, and Cybernetics, Vol. 19, No. 1, 1989, pp. 31-39. doi: $10.1109 / 21.24529$

[29] W.-R. Zhang, S. Chen, W. Wang and R. King, "A Cognitive Map Based Approach to the Coordination of Distrib- uted Cooperative Agents," IEEE Transactions on Systems, Man, and Cybernetics, Vol. 22, No. 1, 1992, pp. 103-114. doi:10.1109/21.141315

[30] Fermi National Accelerator Laboratory, Press Room 06-19, 25 September 2006.

[31] W.-R. Zhang, A. Pandurangi and K. Peace, "YinYang Dynamic Neurobiological Modeling and Diagnostic Analysis of Major Depressive and Bipolar Disorders," IEEE Transactions on Biomedical Engineering, Vol. 54, No. 10, 2007, pp. 1729-1739. doi:10.1109/TBME.2007.894832

[32] W.-R., Zhang, K. A. Pandurangi, K. E. Peace, Y. Zhang and Z. Zhao, "MentalSquares: A Generic Bipolar Support Vector Machine for Psychiatric Disorder Classification, Diagnostic Analysis and Neurobiological Data Mining," International Journal on Data Mining and Bioinformatics, Vol. 17, No. 4, 2011, pp. 547-576.

[33] W.-R. Zhang, "YinYang Bipolar Fuzzy Sets and Fuzzy Equilibrium Relations for Bipolar Clustering, Optimization, and Global Regulation," International Journal of Information Technology and Decision Making, Vol. 5, No. 1, 2006, pp. 19-46.

[34] A. Einstein,"Considerations Concerning the Fundaments of Theoretical Physics," Science, Vol. 91, No. 2369, 1940, pp. 487-491. doi:10.1126/science.91.2369.487

[35] P. Shor, "Algorithms for Quantum Computation: Discrete Log and Factoring," Proceedings of 35th Annual Symposium on Foundation of Computer Science, IEEE Computer Society, Los Alamitos, 1994, pp. 124-134.

[36] G. Birkhoff and J. von Neumann, "The Logic of Quantum Mechanics," Annals of Mathematics, Vol. 37, No. 4, 1936, pp. 823-843. doi:10.2307/1968621

[37] P. Woit, "Not Even Wrong: The Failure of String Theory and the Search for Unity in Physical Law," Basic Book, New York, 2006.

[38] L. A. Zadeh, "Fuzzy Logic," Scholarpedia, Vol. 3, No. 3, 2008, p. 1766. 